\title{
The use of selected neutron absorption resonance filters to suppress spurious events on hot neutron spectrometers
}

\author{
D. Lançon ${ }^{\mathrm{a}, \mathrm{c}, *}$, R.A. Ewings ${ }^{\mathrm{b}}$, J.R. Stewart ${ }^{\mathrm{b}}$, M. Jiménez-Ruiz ${ }^{\mathrm{c}}$, H.M. Rønnow ${ }^{\mathrm{a}}$ \\ a Laboratory for Quantum Magnetism, Ecole Polytechnique Fédérale de Lausanne (EPFL), 1015 Lausanne, Switzerland \\ ${ }^{\mathrm{b}}$ ISIS Facility, STFC Rutherford Appleton Laboratory, Didcot OX11 0QX, United Kingdom \\ ${ }^{\mathrm{C}}$ Institut Laue-Langevin, BP156, 38042 Grenoble Cedex, France
}

\section{A R T I C L E I N F O}

Article history:

Received 10 August 2014

Received in revised form

1 December 2014

Accepted 31 December 2014

Available online 12 January 2015

Keywords:

Neutron resonance filter

Neutron absorption cross-section

Hot neutron spectroscopy

\begin{abstract}
A B S T R A C T
Resonant absorption can be used as a filter for high energy neutron spectroscopy. Here we report the transmission of eight thin foil filters: erbium, indium, iridium, dysprosium, hafnium, gadolinium, cadmium and samarium, measured using neutron time-of-flight techniques over a range of energies ( $1 \mathrm{meV}$ to $10 \mathrm{eV}$ ). Measured transmission is converted into energy-dependent absorption cross-section which compares closely to tabulated values. Each resonance is characterized from $91 \mathrm{meV}$ (samarium) to $2815 \mathrm{meV}$ (gadolinium) by Lorentzian fits. Possibilities for the use of neutron filters depending on the type of spurious background are discussed and the performance is simulated for a specific example of a hot neutron triple axis spectrometer experiment.
\end{abstract}

(c) 2015 Elsevier B.V. All rights reserved.

\section{Introduction}

Neutron absorption filters can be used in hot neutron experiments in order to reduce spurious signals and background by filtering out unwanted neutron energies. This is especially important on three-axis spectrometers, such as the hot neutron triple axis IN1 at the ILL, as these instruments are prone to spurious signals [1]. Hot neutron triple-axis spectroscopy may for instance be a method to measure high-energy spin-wave dispersions in undoped and doped cuprate superconductors [2]. Renewed interest in such measurements comes from the establishment of quantitative Hubbard projection predictions [3,4], the discovery of a zone-boundary line-shape quantum anomaly [5,6], and the recent observation of relatively well-defined dispersion even at optimal doping [7]. In particular in hot neutron experiments, measurements are challenged by spurious signals which can be reduced using filters $[1,8]$. Materials widely used as filters are typically erbium for removal of second order harmonics and cadmium to stop thermal neutrons [9], although hot neutron spectroscopy can be further improved using a broader range of resonance filters.

Presented here are systematic comparative transmission measurements of eight neutron resonance filters: erbium, indium, iridium, dysprosium, hafnium, gadolinium, cadmium and samarium. We obtained reliable transmission data over the range of neutron

\footnotetext{
* Corresponding author at: Laboratory for Quantum Magnetism, Ecole Polytechnique Fédérale de Lausanne (EPFL), 1015 Lausanne, Switzerland.

E-mail address: diane.lancon@epfl.ch (D. Lançon).
}

energies of $10 \mathrm{meV}$ to $10 \mathrm{eV}$, that are shown to verify calculated cross-section from a database [10]. This energy range covers the incident energies of usable thermal and hot neutrons for spectrometry from bound scattering centers. A time-of-flight approach was particularly suited for these measurements, as it allows simultaneous measurement of each filter transmission for the full available neutron energy spectrum without changing angles. The analysis of the transmission curves yields precise information on each material's resonance energies and other characteristics such as efficiency and selectivity. Various filter uses are detailed and an instrument simulation is presented to show spurious signals removed using filters. This gives insights into the design of a thermal or hot neutron experiment that would require filtering and provide guidance in the choice of a filter adapted to the desired experimental energy range.

\section{Transmission measurements}

The neutron transmission measurements were carried out at the pulsed spallation neutron source ISIS [11] (Rutherford Appleton laboratory) on the ALF beam-line.

The samples consisted of thin foils of each filter element (with high purity, $97 \%$ for Hf, 99.9\% for all others) placed on the direct beam path, and measured at room temperature. The filters available as samples had the following thicknesses $d$ : Gd $0.1 \mathrm{~mm}$, In $0.1 \mathrm{~mm}$, Ir $0.1 \mathrm{~mm}$, Cd $0.1 \mathrm{~mm}$, Er $0.1 \mathrm{~mm}$, Dy $0.2 \mathrm{~mm}$, Sm $0.25 \mathrm{~mm}$ and Hf $0.25 \mathrm{~mm}$. 
Neutron energies are obtained from their time of flight, and in the experimental configuration of ALF, the total flight path length from the moderator to the monitor was $15.97 \mathrm{~m}$, with the foils placed at $2.35 \mathrm{~m}$ from the detector. The chopper was synchronized in order to access energies up to $10 \mathrm{eV}$. The detectors were continuous neutron beam monitors using ${ }^{6} \mathrm{Li}$ containing scintillating glass of thickness $0.25 \mathrm{~mm}$, along with a $38 \mathrm{~mm}$ photomultiplier tube for light collection, yielding a $1 \%$ efficiency at $k=$ $6.28 \AA^{-1}$. For statistical confidence, data was accumulated for each filter (and open beam ) for $\sim 30 \mathrm{~min}$ (total integrated proton beam current $\sim 70 \mu \mathrm{A} \mathrm{h})$.

The transmission of filters was obtained by dividing the energydependent count rate with a corresponding measurement performed without any filter in the beam. The beam size was $16.5 \mathrm{~cm}^{2}$; except in the case of iridium where it was reduced to $4 \mathrm{~cm}^{2}$ by a Boron-containing diaphragm to accommodate the small sample size. The thickness of the diaphragm was sufficient to remove neutrons up to at least $10 \mathrm{eV}$.

\section{Results}

The measured transmissions of each filter element for energies up to $10 \mathrm{eV}$ are plotted in Fig. 1 as a function of both neutron wavenumber $k$ and neutron energy $E=2.071\left[\mathrm{meVA}^{2}\right] \mathrm{k}^{2}$ (raw data accessible from [12]). The observed abrupt reductions of transmission at particular wavenumbers are typical characteristics of neutron absorption resonances.

For comparison, data obtained from the Evaluated Nuclear Data File database (ENDF) [10] is also plotted in Fig. 1. Tabulated neutron absorption cross-sections for each isotope of the filter elements were averaged by isotope abundances to yield the absorption crosssection $\sigma_{\text {abs. }}$. The transmission $T$ was then obtained, as $T(E)=$ $\exp (-\mu(E) \cdot d)$ with $d$ being the sample thickness and $\mu$ being the linear absorption coefficient given by $\mu=\rho N_{a} / M \sigma_{a b s}$, where $\rho$ is the density, $M$ is the atomic molar mass and $N_{a}$ is the Avogadro number. We provide for download a matlab program to calculate transmission based on the ENDF database [13]. The overall agreement between the measurements and the database is very clear from the transmission curve, as both the position and the width of the absorption peaks are reproduced. Comparing our dataset with the ENDF database, it can be concluded that the tabulated data of the cross-section are applicable for real filters made of commercially available foils.

To remove the thickness dependency from the filter data, the transmission of each filter was converted into absorption crosssection as a function of neutron energy. Lorentzian functions reproduce very closely the measured absorption resonance peaks' profiles and the characteristics of each absorption resonance peak could thus be extracted from a Lorentzian fit of the absorption peak. Such analysis was performed for all resonances up to $3 \mathrm{eV}$, as higher energy filtering should not be required, based on the accessible neutron spectrum of current neutron sources.

Table 1 shows each material's characteristics: the absorption resonance energy values $E_{\text {res }}$ and cross-section $\sigma_{a b s}\left(E_{r e s}\right)$, along with the full width half maximum (FWHM) of each absorption resonance and $\sigma_{2200}$, the thermal cross-section from tabulated absorption cross-section values [14] for $2200 \mathrm{~m} / \mathrm{s}$ neutrons $\left(k_{2200}=3.495 \AA^{-1}\right)$.

As the total cross-section of low energy neutrons was also accessible in our dataset, this allows estimation of their efficiency in filtering out thermal neutrons. Absorption cross-section for low energy neutrons usually increases linearly with $\lambda$ and is thus given
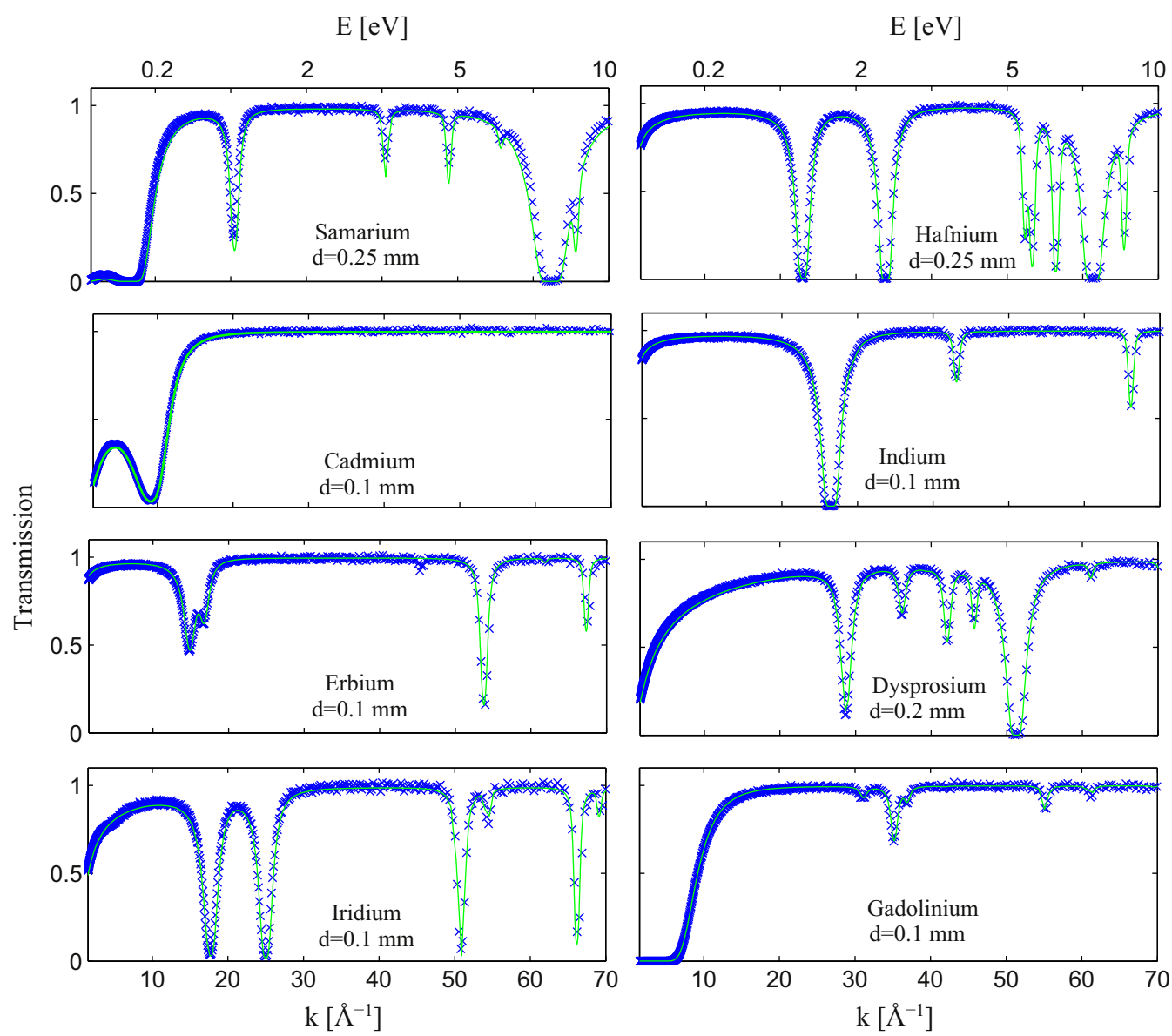

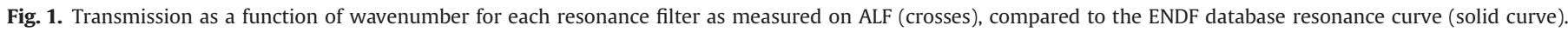


by $\sigma_{2200} \cdot\left(k_{2200} / k\right)$. Adolinium, samarium and cadmium have the strongest thermal neutron absorption cross-section (respectively $\sigma_{2200}=49700$ barns, 5922 barns and 2520 barns), making them efficient thermal neutron absorbers. This characteristic is visible on the transmission curve, where low transmission values are reached at small wavenumbers (below $10 \AA^{-1}$ ). Dysprosium, iridium, hafnium, indium and erbium have lower absorption cross-section (Table 1) for neutrons with energies lower than $500 \mathrm{meV}$, so that large decreases in transmission only occur at the measured resonances.

As a side result of looking at these data we point out some effects about Cd which should be taken into account when used as a shielding material in neutron instrumentation. Depending on application, typically $\mathrm{Cd}, \mathrm{Gd}, \mathrm{B}$ and $\mathrm{Li}$ are used for neutron shielding, and part of the materials choice relates to the absorption cross-section. However, using only the tabulated values for $2200 \mathrm{~m} / \mathrm{s}$ neutrons gives an incomplete picture of Cd's performance, as illustrated in Fig. 2. Despite Gd having a 20 times higher tabulated cross-section, the $164 \mathrm{meV}$ resonance makes Cd more efficient in the 110-480 meV range for the same thickness. Conversely, the tail of the $164 \mathrm{meV}$ resonance reaches past $k_{2200}$, meaning that using the tabulated value over-estimates the absorption of Cd for wave-vectors below $3 \AA^{-1}(<20 \mathrm{meV})$, below which one should rather use the value $1495 \mathrm{barn} \cdot\left(k_{2200} / k\right)$. As can be

Table 1

From ALF experiment: filters' absorption resonance energies $E_{\text {res }}$ up to $3 \mathrm{eV}$ with absorption cross-section at the resonance $\sigma_{a b s}\left(E_{\text {res }}\right)$, the full width at half maximum FWHM and tabulated thermal absorption cross-section $\sigma_{2200}$ (barn) from [14].

\begin{tabular}{lllll}
\hline Filter & $\sigma_{2200}(\mathrm{barn})$ & $E_{\text {res }}(\mathrm{meV})$ & $\sigma_{a b s}\left(E_{\text {res }}\right)(\mathrm{barn})$ & FWHM (meV) \\
\hline Sm & 5922 & 91 & 11750 & 59 \\
Sm & & 861 & 1936 & 80 \\
Cd & $2520^{\text {a }}$ & 164 & 7204 & 131 \\
Er & 159 & 454 & 2278 & 126 \\
Er & & 578 & 1475 & 99 \\
Ir & 425 & 646 & 4764 & 96 \\
Ir & & 1291 & 5086 & 113 \\
Hf & 104 & 1088 & 4994 & 96 \\
Hf & & 2371 & 5419 & 120 \\
In & 193 & 1447 & 18670 & 113 \\
Dy & 994 & 1706 & 3588 & 132 \\
Dy & & 2705 & 625 & 150 \\
Gd & 49700 & 1991 & 236 & 153 \\
Gd & & 2558 & 1310 & 88 \\
Gd & & 2815 & 356 & \\
\hline
\end{tabular}

${ }^{\mathrm{a}}$ For $\sigma_{2200}$ of cadmium, see discussion of Fig. 2.

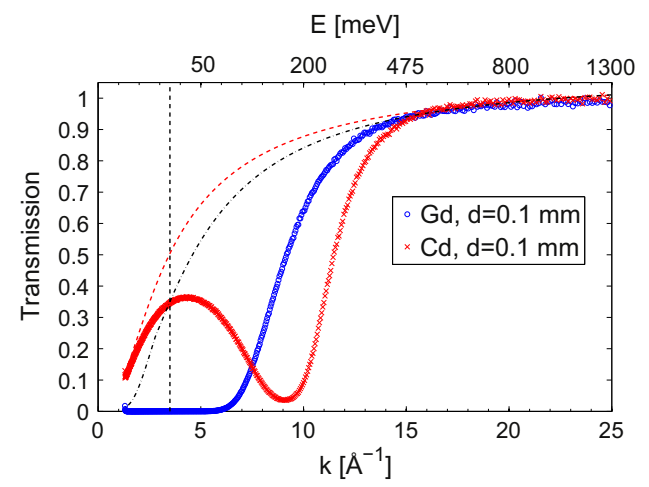

Fig. 2. Comparison of the transmission of gadolinium (circles) and cadmium (crosses), for the same filter thickness $d=0.1 \mathrm{~mm}$. The vertical line signals $k_{2200}=3.495 \AA^{-1}$ where thermal absorption cross-sections are normally given. Dashed red line indicates the transmission cadmium that would have without the $164 \mathrm{meV}$ resonance and the dash-dotted black line indicates the thermal transmission calculated using the tabulated $\sigma_{2200}$. (For interpretation of the references to color in this figure caption, the reader is referred to the web version of this paper.)

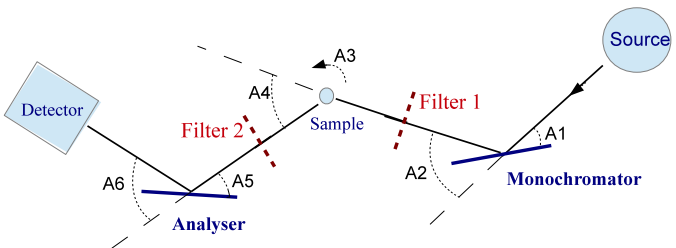

Fig. 3. A diagram of a triple axis spectrometer with possible filter position, using IN1 configuration.

seen in Fig. 2 the difference in transmission e.g. at $5 \mathrm{meV}$ is a factor of 5.2. Energy dependant cross-sections should thus be used for a more accurate estimation of the efficiency of filters [15].

\section{Uses of filters}

We discuss the different possible uses of filters in the design and optimization of thermal or hot neutron experiments. While this is particularly useful in the case of a triple axis spectrometer, experiments on angle-dispersive diffractometers can also benefit from filter optimization.

In a neutron inelastic experiment, the crucial parameters are the incident and outgoing energies $E_{i}$ and $E_{f}$, with the resulting energy transfer $\Delta E=E_{i}-E_{f}$. For a triple axis spectrometer (Fig. 3), spurious events can come from the following three elements: the monochromator, the analyzer and the materials located at the sample position. All three can scatter strongly at a Bragg peak position at the desired energy as well as at the higher order harmonics and create weaker backgrounds due to incoherent scattering. The hierarchy of such spurious events is as follows:

1. The strongest event comes from Bragg scattering at all three elements. A small rotation of the sample would identify and eliminate this event.

2. The second strongest spurious events come from two strong Bragg scattering and weak incoherent scattering at the third element. In the case of the Bragg scattering at the sample and either monochromator or analyzer, a sample rotation also removes this event. However, the case of incoherent scattering at the sample and Bragg scattering at analyzer and monochromator cannot be removed by sample rotation. For each $n^{2} E_{i}=m^{2} E_{f}$, with $m, n=1,2,3$ there is thus a spurious event, particularly strong when $E_{i}=4 E_{f}$, also known as a $\lambda / 2$ contamination.

3. Weaker spurious signals further come from two incoherent events, and one strong Bragg scattering event at either $m^{2} E_{f}$ at the analyzer or $n^{2} E_{i}$ at the monochromator. This can be removed using a resonance filter used as a notch filter, i.e. a narrow band stop filter, placed before the sample to remove $n^{2} E_{i}$ or after the sample to remove $m^{2} E_{f}$.

For all spurious signal for which a resonance filter can be used, the thickness of the filter chosen is a crucial parameter in the design of an experiment. The following sections will show how to choose a resonance filter as a $\lambda / 2$ filter (removing $E_{i}=4 E_{f}$ ) as well as a notch filter to remove the incoherent spurious events, followed by a description of thickness optimization and an example of the use of filters in order to optimize a hot neutron triple axis experiment.

\subsection{Use of $a \lambda / 2$ filter}

As the spurious event corresponding to $E_{i}=4 E_{f}$ is particularly strong, it can be necessary to use a $\lambda / 2$ filter. Its choice requires knowledge of the characteristics of the absorption resonance 
Table 2

For a filter thickness of $d=0.1 \mathrm{~mm}$ : absorption resonance energy $E_{\text {res }}$ and working energy $E_{\text {exp }}=E_{\text {res }} / 4$ with their respective transmission $T$ and the absorption ratio $\sigma_{a b s}\left(E_{\text {res }}\right) / \sigma_{a b s}\left(E_{\text {exp }}\right)$.

\begin{tabular}{lllllc}
\hline Filter & $E_{\text {res }}(\mathrm{meV})$ & $T$ & $E_{\text {exp }}(\mathrm{meV})$ & $T$ & $\frac{\sigma_{a b s}\left(E_{\text {res }}\right)}{\sigma_{a b s}\left(E_{\text {exp }}\right)}$ \\
\hline Er & 454 & 0.47 & 113.5 & 0.96 & 16.4 \\
Er & 578 & 0.62 & 144.5 & 0.96 & 9.9 \\
Ir & 646 & 0.03 & 161.5 & 0.88 & 26.8 \\
Ir & 1291 & 0.01 & 323 & 0.88 & 36 \\
Hf & 1088 & 0.10 & 272 & 0.98 & 94.8 \\
Hf & 2371 & 0.01 & 593 & 0.97 & 96.7 \\
In & 1447 & $2 \cdot 10^{-5}$ & 362 & 0.97 & 201 \\
Dy & 1706 & 0.42 & 426.5 & 0.93 & 14.6 \\
\hline
\end{tabular}

peaks of each material, since $4 E_{f}$ neutrons should be filtered out without removing neutrons with energy $E_{f}$.

Table 2 shows a list of potentially useful $\lambda / 2$ filters with their absorption resonance energy value $E_{\text {res }}$ as well as the energy $E_{\text {exp }}$ $\left(E_{\text {res }} / 4\right)$, which corresponds to the particular energy one should work within an experiment in combination with such a filter. For example, when performing an experiment with hot neutrons around $150 \mathrm{meV}$, the choice of a particular neutron energy such as $144.5 \mathrm{meV}$ or $161.5 \mathrm{meV}$ allows the use of respectively erbium or iridium filters to remove $\lambda / 2$ neutrons. Table 2 also gives the transmission of such filters at $E_{\text {exp }}$ and $E_{\text {res }}$ for a filter thickness of $d=0.1 \mathrm{~mm}$.

The absorption ratio $\sigma_{a b s}\left(E_{\text {res }}\right) / \sigma_{a b s}\left(E_{\text {exp }}\right)$ given in Table 2 provides information on the efficiency of a filter, and large ratios should be favored in order to remove unwanted energies without a significant reduction of the intensity at the experimental energy. In that respect, the indium filter is the best, with a very high absorption ratio, followed by hafnium with half the indium ratio. An indium filter $(d=0.1 \mathrm{~mm})$ thus allows $99.99 \% \lambda / 2$ suppression for $97 \%$ transmission of desired neutrons. On the other hand, the low absorption ratio of the resonances of samarium limits its use as a resonance filter as the second resonance allows $98 \% \lambda / 2$ suppression for only $49 \%$ transmission of desired neutrons $(d=0.25 \mathrm{~mm})$. Additional information on the selectivity of the filters can also be obtained from the full width at half maximum of the Lorentzian fits of the absorption peaks (given in Table 1). Broad resonance give additional flexibility in the choice of $E_{\text {exp }}$, allowing to tune $E_{\text {exp }}$ according to experimental constraints other than filtering. One can also select additional filters in order to remove higher order contaminations: $n^{2} E_{i}=m^{2} E_{f}$, with $m, n=1,2,3$.

\subsection{Use of an $E_{i}$ or $E_{f}$ notch filter}

Background due to incoherent scattering at the monochromator and sample followed by coherent scattering at the analyzer will be neutrons with energy $E_{f}$. Such neutrons can be removed by placing an $E_{f}$ notch filter with a resonance at $E_{f}$ before the sample or, if space permits, even before the monochromator. Unlike the $\lambda I$ 2 filters, such a notch filter influences the range of measurable energy transfers $\Delta E$. It will no longer be possible to measure close to zero energy transfer since, in that case, $E_{f}=E_{i}$ and thus such neutrons are removed by the filter.

Conversely, background due to coherent scattering at the monochromator followed by incoherent scattering at the sample and the analyzer will be neutrons with energy $E_{i}$, which can be removed by placing an $E_{i}$ notch filter with a resonance at $E_{i}$ after the sample. If the filter material has a sizable low-energy absorption this will place a lower limit on measurable $E_{f \text { min }}$. For energy scans where $E_{i}$ is kept fixed at the filter resonance, the measurable energy range is hence limited to FWHM $<\Delta E<E_{i}-E_{f} \min$. However, for resolution and flux reasons, TAS energy scans are normally conducted with fixed $E_{f}$ and varying $E_{i}$. In that case an $E_{i}$ notch filter limits the accessible energy range to the width of the resonance.

In the case of iridium, the resonance corresponding to $E_{i}=$ $646 \mathrm{meV}$ can be used to suppress incoherent scattering. Using an iridium filter to reduce detected neutrons with energy $E_{i}$ by a factor 100 , the suited range of $E_{f}$ for a transmission above $80 \%$ is $81<E_{f}<427 \mathrm{meV}$, which corresponds to energy transfers from $219 \mathrm{meV}$ up to $565 \mathrm{meV}$. The thermal absorption cross-section is sufficiently low to allow very high energy transfer. However, in the case of dysprosium or gadolinium, for example, their high thermal absorption ratio would limit the available energy transfer when they are used as a notch filter. To choose an efficient notch filter, the limit on the range of energy transfer should thus be estimated using the filter's transmission data. Table 3 shows potential notch filters and their range of energy transfers with $80 \%$ transmission among the eight filters measured, for each filter's recommended thickness.

\subsection{Thickness optimization}

The transmission depends exponentially on the thickness of the filter and thus one can optimize the filter efficiency by varying this parameter. If the thickness is changed from the given reference $x_{0}$ with transmission $T_{0}$ to $x$, the new transmission is given by $T(x)=T_{0}^{\left(x / x_{0}\right)}$

Erbium is a typical case where thickness considerations are needed, as the absorption ratios $\sigma_{a b s}\left(E_{\text {res }}\right) / \sigma_{a b s}\left(E_{\text {exp }}\right)$ of $454 \mathrm{meV}$ and $578 \mathrm{meV}$ resonances are low. With an erbium filter of $0.1 \mathrm{~mm}$ thickness, the suppression at $454 \mathrm{meV}$ resonance is $50 \%$, with an intensity suppression of $\sim 4 \%$ at $E_{\exp }$ (Fig. 4 and Table 2). By doubling the thickness of such a filter, the intensity at the resonance is suppressed by $77 \%$ while at $E_{\text {exp }}$ the intensity is reduced by $7 \%$. In order to obtain almost complete filtering at the resonance, an erbium filter of $0.4 \mathrm{~mm}$ can be used to suppress $97 \%$ of the intensity at the resonance while causing a mild reduction of the intensity at $E_{\exp }$ of $14 \%$.

A further effect of increasing the thickness is the broadening of the dip in transmission, which can be desirable in order to filter out energies away from the resonance energies, e.g. if used as an $E_{i}$ notch filter with fixed $E_{f}$. Indium's very high absorption ratio, which reduces transmission at the resonance to effectively zero with a filter of $0.1 \mathrm{~mm}$, allows this material to demonstrate the effect. Upon the increase of the filter thickness, the dip in the transmission curve broadens (Fig. 4). An In filter of $0.4 \mathrm{~mm}$ instead of $0.1 \mathrm{~mm}$ increases the full-width half-maximum by $105 \%$, while causing only a $9 \%$ reduction of transmission at $E_{\text {exp }}$. For Er, using a relatively thick $0.8 \mathrm{~mm}$ filter, the double resonance provides $<5 \%$ transmission in the entire interval $400-600 \mathrm{meV}$ albeit at the cost of only $60-70 \%$ transmission in the $50-250 \mathrm{meV}$ range.

\section{Table 3}

Examples of $E_{i}$ notch filters with variable $E_{f}$ : recommended thickness $d$ in $\mathrm{mm}$ for each filter, resonance energy $E_{\text {res }}$ chosen as $E_{i}$ with its transmission $T$ and the range of energy transfer with transmission $T \geq 80 \%$.

\begin{tabular}{lllll}
\hline Filter & $\begin{array}{l}\text { Recommended thickness } \\
(\mathrm{mm})\end{array}$ & $\begin{array}{l}E_{\text {res }}=E_{i} \\
(\mathrm{meV})\end{array}$ & $T$ & $\begin{array}{l}\Delta E(\mathrm{meV}) \text { with } \\
T \geq 80 \%\end{array}$ \\
\hline $\mathrm{Er}$ & 0.3 & 454 & 0.11 & $163-437$ \\
$\mathrm{Er}$ & 0.3 & 578 & 0.24 & $287-561$ \\
$\mathrm{Ir}$ & 0.1 & 646 & 0.03 & $168-600$ \\
$\mathrm{Ir}$ & 0.1 & 1291 & 0.01 & $200-488$ \\
$\mathrm{Sm}$ & 0.5 & 861 & 0.07 & $143-524$ \\
$\mathrm{Hf}$ & 0.1 & 1088 & 0.10 & $126-E_{i}$ \\
$\mathrm{In}$ & 0.1 & 1447 & $2 \times 10^{-5}$ & $330-E_{i}$ \\
\hline
\end{tabular}



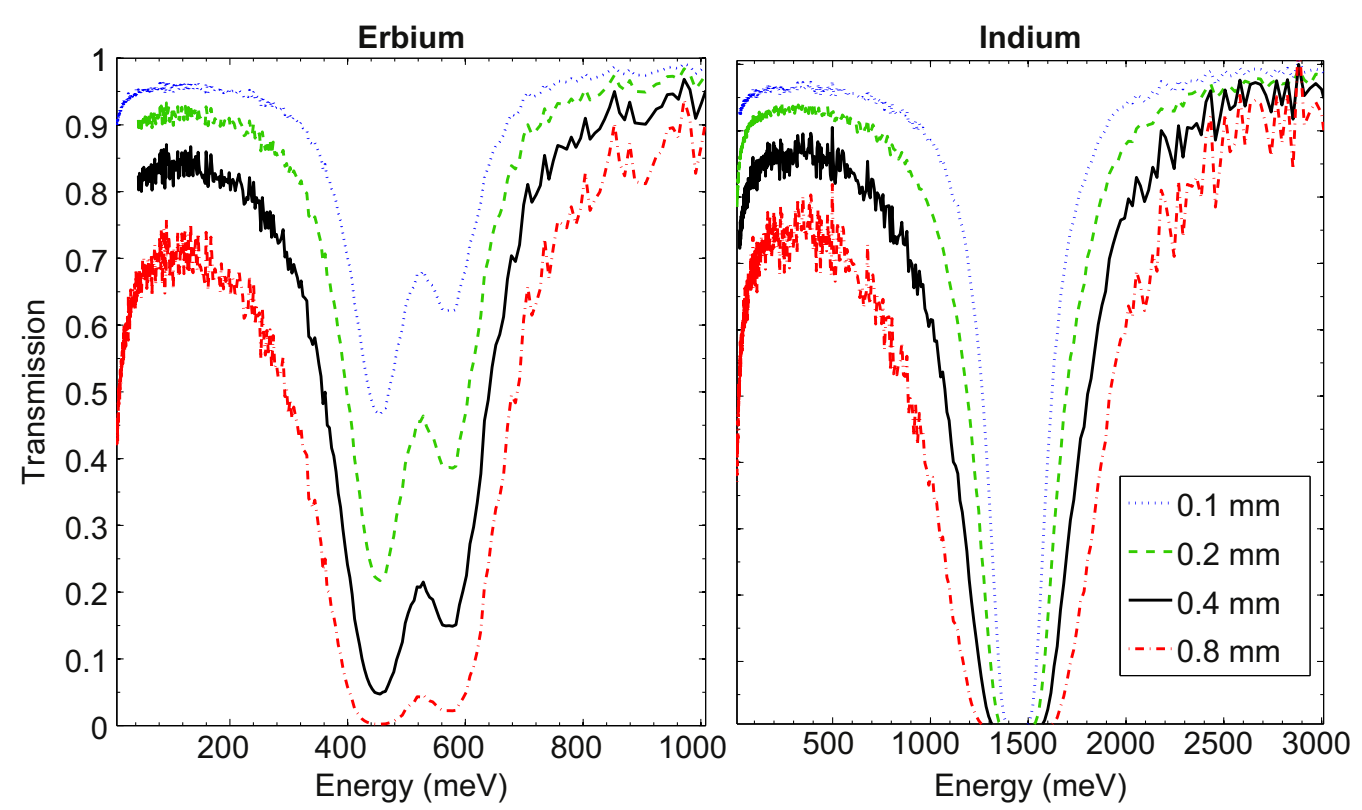

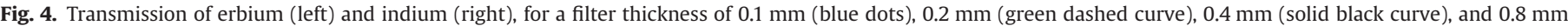

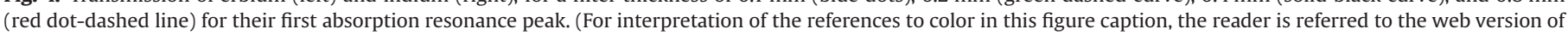
this paper.)
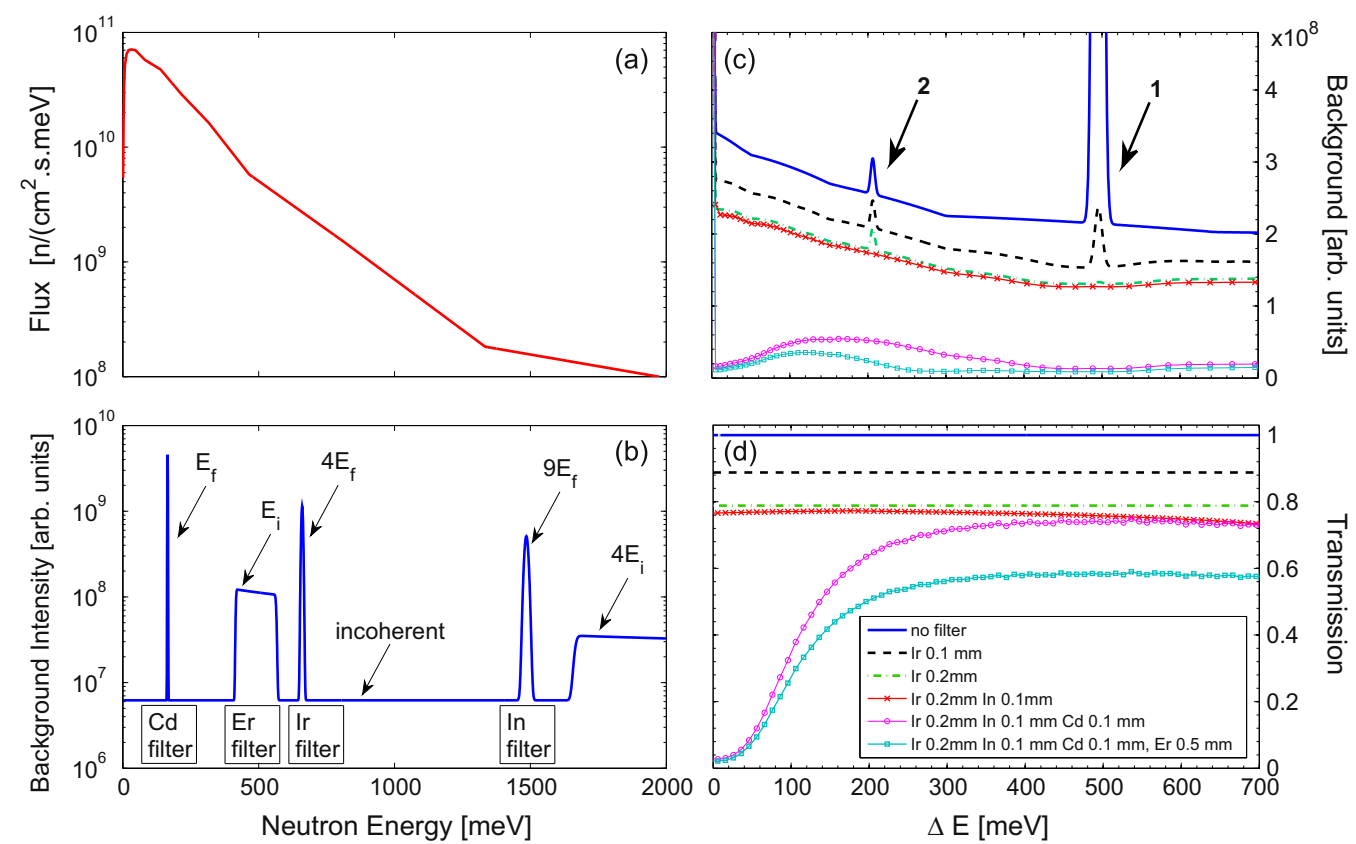

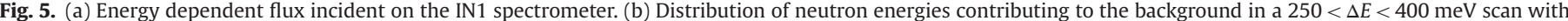

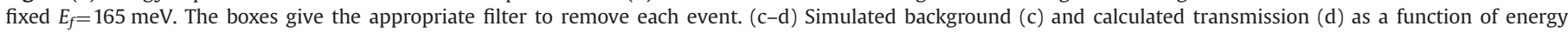

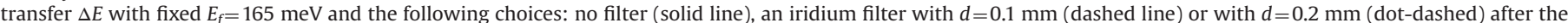

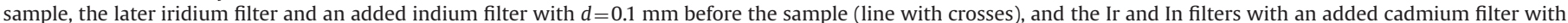

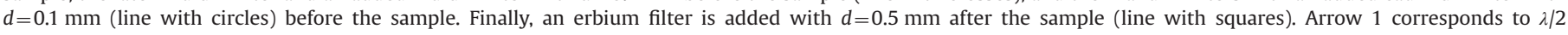
contamination $\left(E_{i}=4 E_{f}\right)$ while arrow 2 corresponds to the higher order contamination $4 E_{i}=9 E_{f}$.

\subsection{Filters for an experiment on the hot neutron triple axis spectrometer IN1}

To demonstrate how resonance filters may be combined to remove spurious signals, we describe the case of a hot triple axis experiment where the desired energy transfer is around $300 \mathrm{meV}$ (zone boundary of spin waves in cuprates [2]). The background and the transmission as a function of $E_{i}$ and $E_{f}$ were simulated using the instrumental characteristics and flux of the hot neutron triple axis spectrometer IN1 at the ILL, which has a Cu(331) monochromator and a $\mathrm{Cu}(200)$ analyzer. The transmission functions of the monochromator and analyzer were approximated by the following function:

$T\left(E_{b}, E\right)=S_{\text {inc }}+\left(1-S_{\text {inc }}\right) \sum_{n=1}^{n=\infty} R_{n} \frac{1}{\sqrt{\sigma}} \exp \left(-\frac{1}{2} \frac{\left(n^{2} E_{b}-E\right)^{2}}{\sigma^{2}}\right)$

with $E_{b}$ being corresponding to either $E_{i}$ or $E_{f}$, the nominal energies to which respectively the monochromator and the analyzer are set, $R_{n}$ being the reflectivity of the $n$th harmonics $(n=1,2,3)$ approximated to $1 / n, \sigma \sim 0.05 \cdot n^{2} \cdot E_{b}$, and $S_{i n c}=1.5 \times 10^{-3}$ the incoherent 
scattering. Incoherent scattering $S_{i n c}$ for the monochromator and the analyzer was estimated using experimental data from IN1 and assumed to be equal. For the sample, only incoherent scattering was taken into account. Combining this with the filter transmission as well as the neutron flux energy dependence (Fig. 5, for the IN1 case), we thus obtained a simulation of the background for any combination of $E_{i}$ and $E_{f}$. This simple parametrization of the transmission function is not quantitatively accurate, but allows us to qualitatively track the sources of background.

The result of this study is presented in Fig. 5 for a fixed $E_{f}=165 \mathrm{meV}$. Fig. 5a shows the energy dependent flux incident on the IN1 spectrometer. Fig. 5b shows the distribution of the neutron energies that contribute to the background for a scan with $250<\Delta E<400 \mathrm{meV}$. Fig. $5 \mathrm{c}$ and d shows the simulated background and calculated transmission for a number of filter combinations. The solid line corresponds to the background and the transmission as a function of energy transfer in the absence of filters. As expected from the hierarchy of spurious events, the background consists of two strong spurious events (type 2) on an incoherent continuous background (type 3). The strongest spurious event (arrow 1, Fig. 5c) at $\Delta E \sim 485\left(E_{i} \sim 650 \mathrm{meV}\right)$ corresponds to the $\lambda / 2$ contamination, $E_{i}=4 E_{f}$. The second strong spurious event (arrow 2, Fig. 5c) at $\Delta E \sim 205\left(E_{i} \sim 370 \mathrm{meV}\right)$ corresponds to the weaker higher order contamination $4 E_{i}=9 E_{f}=1485 \mathrm{meV}$.

$E_{f}=165 \mathrm{meV}$ was chosen such that the $646 \mathrm{meV}$ resonance of Ir could be used to remove the strong $\lambda / 2$ spurion (labelled 1 in Fig. 5), with complete filtering for a thickness $d=0.2 \mathrm{~mm}$ placed after the sample (dot-dashed line). Adding an indium filter of $d=0.1 \mathrm{~mm}$ before the sample removes the second strong spurious event (line with crosses). The combination of these two filters only reduces the transmission of desired neutrons to $>70 \%$ with weak $\Delta E$ variation. A large part of the remaining background can be removed by using the $164 \mathrm{meV} C d$ resonance as an $E_{f}$ notch filter before the sample (line with circles). In that case, the transmission of the desired signal drops dramatically in the $100-200 \mathrm{meV}$ energy range, so that the use of this additional filter is only recommended if the desired $\Delta E$ is above $200 \mathrm{meV}$. If experimental relevant energy transfers are in the range $250<\Delta E<400 \mathrm{meV}$, an additional large fraction of the background can be removed by the double $450-600 \mathrm{meV}$ resonance of a relatively thick erbium $E_{i}$ notch filter $(d=0.5 \mathrm{~mm})$ after the sample (line with squares). The $E_{i}$ notch filter cuts off measurable energy transfers $<200 \mathrm{meV}$, which would be acceptable for example for the zone boundary of cuprates. The transmission is reduced from $70 \%$ to $50 \%$ for a factor of 2-3 reduction in the simulated background in the $250-400 \mathrm{meV}$ energy transfer range.

\section{Conclusion}

In summary we have measured the transmission of eight typical neutron resonance filters. We find actual transmission sufficiently accurately reproduced by calculations based on the ENDF data-base. We discuss typical uses of filters to reject background and illustrate this with a specific example for the hot neutron triple axis spectrometer IN1.

\section{Acknowledgments}

We thank the ISIS and ILL facilities for neutron beam access. We thank D.E. Pooley for assistance with setup of the neutron detectors and A. Ivanov for insightful discussions. The work was supported by the Swiss National Science Foundation.

\section{Appendix A. Supplementary data}

Supplementary data associated with this article can be found in the online version at http://dx.doi.org/10.1016/j.nima.2014.12.114.

\section{References}

[1] G. Shirane, S.M. Shapiro, J.M. Tranquada, Neutron Scattering with a Triple-Axis Spectrometer: Basic Techniques, Cambridge University Press, Cambridge, 2002.

[2] G. Aeppli, S.M. Hayden, H.A. Mook, Z. Fisk, S.-W. Cheong, D. Rytz, J.P. Remeika, G.P. Espinosa, A.S. Cooper, Physical Review Letters 62 (1989) 2052.

[3] B. Dalla Piazza, M. Mourigal, M. Guarise, et al., Physical Review B 85 (2012).

[4] M. Guarise, B. Dalla Piazza, M. Moretti Sala, et al., Physical Review Letters 105 (2010).

[5] H.M. Ronnow, D.F. McMorrow, R. Coldea, et al., Physical Review Letters 87 (2001) 037202.

[6] N.B. Christensen, H.M. Rønnow, D.F. McMorrow, et al., Proceedings of the National Academy of Sciences 104 (2007) 15264.

[7] M. Le Tacon, G. Ghiringhelli, J. Chaloupka, et al., Nature Physics 7 (2011) 725.

[8] D.J. Hughes, B.A. Magurno, M.K. Brussel, Neutron Cross Sections, Brookhaven report BNL, 2nd ed., 325 supplement no. 1, 1960.

[9] B. D'Mellow, D.J. Thomas, M.J. Joyce, P. Kolkowski, N.J. Roberts, S.D. Monk, Nuclear Instruments and Methods in Physics Research Section A: Accelerators, Spectrometers, Detectors and Associated Equipment 577 (2007) 690.

[10] M. Chadwick, M. Herman, P. Oblozinsky, et al., Nuclear Data Sheets 112 (2011) 2887 (Special issue on ENDF/B-VII.1 Library).

[11] G.C. Stirling, ISIS-the UK pulsed spallation neutron source, in: G. Bacon (Ed.), 50 Years of Neutron Diffraction: The Advent of Neutron Scattering1987.

[12] D. Lancon, et al., 1230006, STFC ISIS Facility, 2012. doi:http://dx.doi.org/10. 5286/ISIS.E.24089001.

[13] See supplementary materials or lqm.epfl.ch/page-30969-en.html, 2012.

[14] V. Sears, Neutron News 33 (1992) 26.

[15] ENDF database, 〈https://www-nds.iaea.org/exfor/endf.htm〉, 2014. 